\title{
Transtorno do Desenvolvimento da Coordenação: uma Revisão de LITERATURA $^{1}$ \\ DEVELOPMENTAL COORDINATION DISORDER: A LITERATURE REVIEW
}

\author{
Wagner PULZI ${ }^{2}$ \\ Graciele Massoli RODRIGUES ${ }^{3}$
}

\begin{abstract}
RESUMO: o movimento possui relação direta com a aprendizagem e com as relaçóes sociais. Crianças que apresentam dificuldades de movimento podem possuir o transtorno do desenvolvimento da coordenação (TDC). O presente artigo trata-se de uma revisão bibliográfica sobre o assunto e teve por objetivo discutir o que foi publicado sobre o TDC entre os anos de 2002 e 2012 , apresentando um panorama quanto ao histórico, prevalência, influências na vida cotidiana, acadêmica e social e possibilidades de intervenção. O termo TDC é usado para se referir às dificuldades nas habilidades motoras e que não é devido deficiências de ordem intelectual, sensorial primária ou neurológica. Esse problema afeta a vida das crianças no seu cotidiano, nas atividades de lazer, na escola e na vida social. Na escola, esse transtorno acarreta em baixo rendimento acadêmico, dificuldades de escrita e compreensão de conceitos matemáticos. Na vida social, pode ocorrer o isolamento e a dificuldade de pertencer a um grupo. Tanto os pais como os professores podem auxiliar no desenvolvimento global e social dessas crianças através de estratégias diferenciadas. Atualmente, destaca-se a necessidade de desenvolvimento de propostas que visem desenvolver e potencializar as habilidades dos escolares com dificuldades motoras.
\end{abstract}

PALAVRAS-CHAVE: Educação Especial. Descoordenação Motora. Desenvolvimento Motor.

\begin{abstract}
Movement is directly related to learning and social relationships. Children who have movement difficulties may have developmental coordination disorder (DCD). This article presents a literature review on the subject and aimed to discuss what has been published about DCD between the years 2002 and 2012, with an overview of the history, prevalence, influences in daily life, academic and social relations and possibilities for intervention. The term DCD is used to refer to difficulties in motor skills that are not the result of intellectual, neurological or primary sensory disabilities. This problem affects the lives of children in their daily life, leisure activities, school and social life. At school, this disorder leads to low academic performance, difficulties in writing and understanding of mathematical concepts. In social life, the isolation and the difficulty of belonging to a group may occur. Both parents and teachers can assist in global and social development of children through different strategies. Currently, there is a need for developing proposals designed to develop and enhance the skills of students with motor difficulties.
\end{abstract}

KEYWORDS: Special Education. Motor Discoodination. Motor Development.

\section{INTRODUÇÃo}

O movimento é essencial para o desenvolvimento das crianças, permitindo as interações em ambientes sociais e físicos e favorecendo a aquisição de diferentes tipos de experiências (DECONINCK et al., 2006). A inter-relação entre ação, percepção e cognição é exigida quando exploramos e monitoramos o ambiente; quando exploramos o espaço, resolvemos problemas motores, expressamos nossas ideias e nos relacionamos com as pessoas. $\mathrm{O}$ movimento contribui com nossa eficiência nos domínios da percepção e cognição, tendo implicações também na esfera social (LARKIN; SUMMERS, 2004).

\footnotetext{
${ }^{1}$ http://dx.doi.org/10.1590/S1413-65382115000300009

${ }^{2}$ Mestrando em Educação Física pela Universidade São Judas Tadeu, São Paulo, SP, Brasil. wagpul@yahoo.com.br

${ }^{3}$ Programa de Pós-Graduação em Educação Física e Ciências do Envelhecimento da Universidade São Judas Tadeu, São Paulo, SP, Brasil. prof.graciele@usjt.br
} 
Contudo, durante o curso do desenvolvimento motor da criança, há a possibilidade do surgimento de disfunçóes que podem ser reconhecidas pelos pais e/ou professores, especialmente professores de educação física. Crianças que demonstrem tais disfunções motoras podem ter Transtorno do Desenvolvimento da Coordenação (TDC).

O termo TDC, no Brasil ainda é pouco estudado e crianças com problemas de coordenação motora, não têm diagnóstico formal e recebem pouca ou nenhuma assistência especializada (ARAÚJO, 2010). Esse termo é usado para se referir às dificuldades nas habilidades motoras que as crianças possuem e que não é devido deficiências de ordem intelectual, sensorial primária ou neurológica (CERMAK et al., 2002).

Este problema afeta a vida da criança em atividades cotidianas como vestir-se, despir-se, amarrar cadarços de sapatos, abotoar a camisa etc., bem como em atividades físicas como saltos ou driblar com uma bola, tornando essas tarefas extremamente difíceis. Frequentemente apresentam falta de equilíbrio, ritmo, orientação espacial e coordenação motora, influenciando negativamente também em sua vida acadêmica e social (CERMAK et al., 2002; SCHOEMAKER et al., 2003; MON-WILLIAMS et al., 2005; DECONINCK et al., 2006; DECONINCK et al., 2007; JOHNSON; WADE, 2007; RIVARD et al., 2007; FILIPCIC; OZBIC, 2008; FAUGHT et al., 2008; EMCK, et al., 2009; ZWICKER et al., 2009; HILLIER, 2010; COETZEE; PIENAAR, 2010).

Várias limitações nas atividades têm sido documentadas em crianças com TDC. No jogo elas podem se destacar de seus pares por não serem capazes de andar de bicicleta ou participar de ativdades com bola. $\mathrm{Na}$ escola, elas são muitas vezes referidas durante as séries iniciais pelas dificuldades na percepção visual, leitura e escrita, com atividades que solicitem a coordenação motora fina, tais como desenhar e recortar, ou através das habilidades de coordenação motora grossa, tais como as envolvidas no esporte, formando um grupo heterogêneo e as limitaçóes que experimentam são bastante variadas (POLATAJKO; CANTIN, 2006), podendo ainda apresentar déficits na coordenação visomotora (CLARK et al., 2005; WUANG et al., 2008), na pronunciação de palavras (IVERSEN et al., 2005) e com possibilidade de persistência durante a adolescência e idade adulta (IVERSEN, 2005; ZWICKER et al., 2009).

Ainda para Polatajko e Cantin (2006), as dificuldades motoras em crianças com TDC podem ser exemplificadas, tais como as que são identificadas em casa ao vestir-se, colocar meias, usar prendedores, utilizar o zíper, calçar sapatos/botas, amarrar cadarços, utilizar utensílios, tomar banho, lavar o cabelo; na escola, por intermédio da escrita ou desenho que desenvolve-se vagarosamente ou de forma bagunçada, no usar tesoura e cola, na habilidade para desenhar (desenhos imaturos), no manuseio do lápis, na performance nas aulas de ginástica e no lazer, apresentando-se de maneira desajeitada na marcha e na corrida, no equilíbrio, no uso de brinquedos de parques, no andar de bicicleta, no patinar, pular corda, praticar esportes e nos atos de arremessar/receber/chutar bolas.

Além do domínio motor e cognitivo, crianças com TDC também podem apresentar problemas emocionais, tais como baixa autoestima, ansiedade e depressão, podendo levá-las a um isolamento social (WILSON, 2005; RIVARD et al., 2007; ZWICKER et al., 2009; ZWICKER et al., 2012). 


\section{2 Овjetivos}

O presente estudo teve por objetivo discutir o que tem sido pesquisado sobre o TDC entre os anos de 2002 e 2012, apresentando um panorama quanto à prevalência, influências na vida cotidiana, acadêmica e social e possibilidades de intervenção.

\section{Desenvolvimento}

Trata-se de uma revisão bibliográfica sistematizada baseada em estudos publicados entre 2002 e 2012 indexados nas bases de dados Medline, Sportdiscus, Portal de Periódicos da Capes, Scielo e Eric, contendo também referências relevantes que abordam o assunto, obtidas em publicaçóes de livros, dissertaçóes e artigos.

Os termos utilizados para busca foram Physical Education (PE); Movement disorders (MD); Developmental coordination disorder (DCD) e Learning disabilities (LD). Como critérios de inclusão, os artigos deveriam possuir o texto completo, terem sido publicados no período em idioma inglês, deveriam apontar relação com a Educação Física em seu conteúdo e focar crianças em idade escolar. Foram excluídos os estudos com fármacos, animais e cuja amostra era formada apenas de crianças com deficiência.

$\mathrm{Na}$ base de dados Medline, realizou-se a combinação dos termos citados anteriormente (PE; MD; DCD e LD) e após a filtragem e excluindo-se os artigos repetidos, restaram 10 produçóes. Na base Spordiscus, seguindo os mesmos procedimentos, 11 artigos compuseram esse estudo. No Portal de Periódico da Capes foram totalizados apenas 8 artigos a partir dos mesmos procedimentos. As bases de dados Scielo e Eric não retornaram resultados.

A partir dos resultados encontrados e da utilização de outros textos auxiliares, foi organizado o estudo versando sobre histórico, definição, prevalência, implicaçóes e possibilidades de intervenção.

\section{Resultados E discussão}

\subsection{Histórico E DEFINIÇÃo}

No começo do século XX já havia níveis de classificação para os diferentes estados de performance motora, despertando o interesse na identificação e intervenção para crianças e adolescentes com problemas motores moderados. Em 1926, Lippitt preocupou-se especialmente com a "coordenação muscular pobre em crianças" e sugeriu que a raiz dessa dificuldade residia numa condição do sistema nervoso que só poderia ser corrigida com treinos e cuidados (CERMAK et al., 2002).

Em 1937, Orton utilizou o termo "falta de jeito" (clumsiness), o qual não era evidente na literatura até o início da década de 1960, momento onde muitos termos passaram a ser usados para descrever as crianças cujas dificuldades motoras interferiam na vida diária (ZWICKER et al., 2012; CERMAK et al., 2002), como por exemplo, síndrome da criança desajeitada (clumsy child syndrome), dispraxia do desenvolvimento (developmental dyspraxia) e disfunção motora perceptual (perceptual motor dysfunction) (ZWICKER et al., 2012), disfunção cerebral mínima (minimal cerebral dysfunction), deficiência de aprendizagem motora (motor learning impairment) e deficiência da coordenação olho-mão (hand-eye coordination 
impairment) (WERNER et al., 2012), inabilidade física (physical awkwardness), disfunção da integração sensorial (sensory integrative dysfunction) (ZWICKER et al., 2012; WERNER et al., 2012), inabilidade congênita (congenital maladroitness) (CERMAK et al., 2002), disfunção neurológica menor (minor neurological dysfunction) e dano cerebral mínimo (minimal brain damage) (MAGALHÂES et al., 2011) e deficiência sensório-motora (sensory-motor impairment) (BONIFACCI, 2004).

Para aprimorar a comunicação e conhecimento entre os médicos e pesquisadores que trabalhavam com crianças "desajeitadas", uma reunião internacional foi realizada em Londres e Ontário, em 1994, para determinar qual a terminologia deveria ser utilizada para descrever estas crianças. Em Londres, o termo TDC (DCD) foi aceito (ZWICKER et al., 2012), sendo definido como uma "deficiência tanto da performance funcional quanto da qualidade do movimento que não é explicado pela idade, intelecto ou qualquer outra condição diagnosticável” (SANGER et al., 2006, p. 2164).

O termo "transtorno do desenvolvimento da coordenação" (developmental coordination disorder) e os critérios de diagnóstico para TDC foram adicionados à terceira edição do DSM (Diagnostic and Statistical Manual of Mental Disorders) e permanecem na $4^{\mathrm{a}}$ edição, publicada pela APA em 2002. Dez anos após a reunião do Consenso de Londres, mais de $50 \%$ de todos os artigos publicados usaram o termo TDC (DCD), mostrando que este termo está ganhando aceitação como a terminologia preferida (ZWICKER et al., 2012). Em 2006, o Consenso de Londres destacou o acordo dos pesquisadores e clínicos internacionais para manter o termo TDC (DCD) como um distúrbio distinto e único. Recentemente, a European Academy of Childhood Disability (EACD) reafirmou os problemas motores de desenvolvimento, bem como os critérios do DSM-IV para definir o transtorno (ZWICKER et al., 2012).

Esse transtorno pode ser reconhecido em crianças que tenham dificuldades de movimento e que não possuam problemas médicos conhecidos ou doenças neurológicas identificadas, afetando a coordenação motora grossa e fina (American Psychiatric Association - APA, 2002). O TDC é atualmente classificado e reconhecido pela bibliografia e comunidade médica através do diagnóstico dos seguintes critérios estabelecidos pela APA (2002, p. 88):

$A$. O desempenho em atividades diárias que exigem coordenação motora está substancialmente abaixo do nível esperado, considerando a idade cronológica e a inteligência medida do indivíduo. O quadro pode manifestar-se por atrasos marcantes em alcançar marcos motores (por ex., caminhar, engatinhar, sentar), propensão a deixar cair coisas, desajeitamento, fraco desempenho nos esportes ou caligrafia insatisfatória.

$B$. A perturbação do Critério A interfere significativamente no rendimento escolar ou nas atividades da vida diária.

C. A perturbaçấo não se deve a uma condição médica geral, por exemplo, paralisia cerebral, hemiplegia ou distrofia muscular, nem satisfaz os critérios para um Transtorno Invasivo do Desenvolvimento.

D. Em presença de Retardo Mental, as dificuldades motoras excedem aquelas associadas com esse transtorno.

No entanto, ainda pode-se encontrar o termo dispraxia do desenvolvimento (developmental dyspraxia) em vários cursos no Reino Unido e nos Estados Unidos, geralmente utilizado por neurologistas, terapeutas ocupacionais e neuropsicologistas para descrever os 
problemas de aprendizagem e planejamento motor em crianças com TDC (CERMAK et al., 2002). Contudo, esse termo pode ser encontrado de forma incorreta como sinônimo de apraxia, condição a qual se dá devido a alguma lesão cerebral. A dispraxia implica num rompimento do desenvolvimento da práxis devido a uma disfunção do sistema somatossensorial, como no caso das dificuldades de aprendizagem (POOLE et al., 1997; GOODGOLD-EDWARDS; CERMAK, 1990; GIBBS et al., 2007).

Já para Sanger et al. (2006), a dispraxia do desenvolvimento pode ser vista em indivíduos com TDC, sendo comumente uma de suas características fundamentais, mas distingue-se deste por não possuir outros critérios que o diagnostiquem como sendo TDC. Dessa forma, um indivíduo com TDC pode ou não ter a dispraxia do desenvolvimento, o que significa que o diagnóstico de TDC não é necessário para se utilizar o termo em questão.

Segundo Sanger et al. (2006), a causa da dispraxia é desconhecida, mas pode estar associada fortemente a um distúrbio de aprendizagem motora, diminuindo a habilidade de aprender pela simples observação e prática, refletindo a diminuição da capacidade de aprendizagem de novas habilidades.

Embora a terminologia varie, o TDC é mais utilizado por profissionais da saúde, profissionais médicos, cientistas do movimento e pesquisadores internacionalmente relacionados (WERNER et al., 2012).

Gibbs et al. (2007), ainda relatam a existência do termo "desordem de atenção e percepção motora" (disorder of attention and motor perception - DAMP), mas o criticam por se tratar de um termo pejorativo principalmente para classificar pacientes com baixa autoestima, corroborando com a ideia que a terminologia ideal deve ser o TDC, uma vez que é a mais encontrada nas pesquisas realizadas e que deve haver um consenso para que um termo seja utilizado como padrão, a fim de facilitar o diagnóstico e o trabalho dos profissionais da área, assim como deve ser compreensível para os pais e as próprias crianças. No entanto, Gibbs et al. (2007) afirmam que os termos TDC e dispraxia são sinônimos (Ibdem).

Crianças com indicativo de TDC também podem apresentar comorbidades frequentes, tais como síndrome de Asperger, transtorno do déficit de atenção e hiperatividade, dificuldade de comunicação, dificuldade de aprendizagem e retardo mental, sendo que, nesse último caso, as dificuldades motoras devem ser superiores às normalmente associadas a esse quadro (POLATAJKO; CANTIN, 2006).

O diagnóstico de TDC deve ser cuidadoso, o que inclui uma revisão da realização de etapas motoras, habilidades de coordenação motora, habilidades sensoriais e um exame físico e neurológico para que não seja confundido com outras condiçóes médicas, tais como paralisia cerebral, acidentes cerebrovasculares, autismo, hemiplegia e distrofia muscular (POLATAJKO; CANTIN, 2006).

\subsection{PrevalêNCIA}

Foi estimado que entre $6 \%$ e $10 \%$ de todas as crianças em idade escolar possuam diagnóstico para TDC (APA, 2002) e 5\% e 15\% segundo Wilson (2005), Lloyd et al. (2006) e Stracker (2011), mas estudos mostram que esse índice pode chegar a 22\% (CERMAK et al., 
2002). No Brasil, um estudo realizado por França (2008) demonstrou que 10,8\% das crianças entre 7 e 8 anos de escolas municipais de Florianópolis, Santa Catarina, apresentaram o TDC, encontrando-se dentro da média estabelecida pela APA (2002). Contudo, Coutinho et al. (2011), ao analisarem crianças entre 6 e 10 anos de escolas públicas de Porto Alegre, Rio Grande do Sul, encontraram 36\% da amostra com TDC, mostrando que esse índice pode ultrapassar os 22\% encontrados por Cermak et al. (2002), sendo um dos distúrbios mais comuns em crianças em idade escolar (WILSON, 2009).

Segundo Barnhart (2003), o TDC atinge mais os meninos do que as meninas (2:1) devido ao fato que a incoordenação motora nos meninos está relacionada à dificuldade de administrá-la em casa e na escola, sendo que a maior incidência de TDC pode ser encontrada em crianças com histórico de dificuldades pré-natais e perinatais.

Com o objetivo de diagnosticar o TDC, muitos instrumentos têm sido utilizados, mas aspectos motivacionais, culturais e ambientais podem influenciar nos resultados, devendo os mesmos serem analisados com cautela. De acordo com os artigos encontrados nessa revisão, os instrumentos mais utilizados nos estudos foram o Movement Assessment Battery for Children (MABC-2) e o Test of Gross Motor Development (TGMD-2).

O MABC, elaborado por Henderson e Sugden (1992), é indicado para a faixa etária entre 3 e 16 anos e avalia três domínios motores (destreza manual, pegar/lançar e equilíbrio). O TGMD-2, elaborado por Ulrich (2000), é indicado para a faixa etária entre 3 e 10 anos e avalia a motricidade global da criança.

No Brasil, ainda podemos encontrar a Escala de Desenvolvimento Motor (EDM) elaborada por Rosa Neto (2002), que visa identificar possíveis dificuldades no plano motor em crianças entre 2 e 11 anos. Avalia as áreas da motricidade fina, global, equilíbrio, esquema corporal, organização espacial, organização temporal e lateralidade. Entretanto, essa escala não faz referência ao TDC, mas sim à coordenação, podendo após uma avaliação ser identificado alguma alteração.

O TDC pode já ser aparente na primeira infância, mas é difícil avaliar crianças de forma confiável antes dos cinco anos, sendo geralmente notadas a partir do ensino fundamental, pois essa condição interfere claramente na performance escolar e nas atividades diárias (GEUZE, 2003).

\subsection{IMPLICAÇÓES}

Crianças com TDC muitas vezes têm dificuldades com leitura, escrita e raciocínio matemático. Alguns dos problemas já foram apresentados anteriormente (problemas de esquema corporal, falta de equilíbrio e coordenação, dificuldades com a orientação espacial e temporal, entre outros). Todas essas habilidades influenciam a capacidade de uma criança em dominar a leitura, escrita e raciocínio matemático. Eles também são um fator importante que determina sucesso escolar (FILIPCIC; OZBIC, 2008). A impossibilidade destas crianças em executar habilidades motoras a um nível de competência igual às mesmas da sua idade é a característica mais importante desse transtorno (WAELVELDE, 2007). 
Tanto crianças com TDC como crianças com suspeita de TDC apresentam dificuldades significativas em leitura, escrita e ortografia em relação a outras crianças, assim como também apresentam problemas significativos na atenção e ajuste psicossocial. As crianças com TDC não são apenas caracterizadas por distúrbios motores, mas também por problemas cognitivos na memória de trabalho viso-espacial, memória de trabalho verbal, aprendizagem de alfabetização e aprendizagem da matemática (CHENG et al., 2011).

Ozbic e Filipcic (2010), afirmam que o TDC pode gerar dificuldades de aprendizagem e de comportamento em relação ao sucesso escolar, gerando problemas na eficiência gráfica (dificuldades motoras, disgrafia, dificuldades na imitação de gestos, dificuldades de orientação espacial no papel), na aritmética (resolução de problemas), na geometria (relaçôes espaciais e problemas em descrever e analisar figuras geométricas, devido à falta de representação mental) e na leitura. Ainda podem ocorrer problemas com a compreensão de instruçóes escritas, figuras ou esquemas, problemas ortográficos, de escrita ou copiando letras individuais.

A escrita, por exemplo, requer que a criança monitore continuamente esse ato com os olhos e nunca se torna completamente automática em uma criança com TDC. Além disso, há dificuldades em alinhar colunas de números e pode existir lentidão em copiar perguntas de matemática a partir de um texto ou da lousa. Este esforço para a escrita reduz a quantidade de atenção da criança diminuindo a compreensão do conceito. Dificuldades semelhantes são encontradas na ortografia, na escrita de uma carta ou na compreensão das palavras de forma adequada (MISSIUNA et al., 2004).

As dificuldades provocadas pelo TDC também afetam a vida social das crianças. A participação em atividades típicas da infância é essencial ao desenvolvimento da criança, mas a incapacidade de participar dessas atividades pode levá-la à marginalização e ao isolamento social, diminuindo a habilidade da criança em manter suas amizades ou fazer parte de grupos (MANDICH; POLATAJKO, 2003).

Para as crianças que têm dificuldades de coordenação, a participação em atividades escolares que exijam um componente motor requer um grande esforço e, muitas vezes, não há sucesso. Essas dificuldades também têm um impacto sobre a vida da criança para além da sala de aula, pois as crianças com TDC têm dificuldades em se preparar para o recesso, interagindo com seus pares em outras atividades de lazer. $\mathrm{O}$ fracasso precoce e contínuo leva ao baixo rendimento escolar, os sentimentos de autoestima, de abstinência, problemas de comportamento e, em muitos casos, a depressão (MISSIUNA et al., 2004).

Quando as crianças com TDC participam de atividades esportivas, geralmente preferem as atividades individuais, como nadar ou andar de bicicleta. Alguns pais relatam que as principais dificuldades para os filhos nos esportes coletivos incluem a dificuldade de seguirem as regras e a orientação espacial, chegando ao ponto de não saberem a direção que devem correr (MAY-BENSON et al., 2002).

As interações são interdependentes e recíprocas, dependentes da resposta motora das crianças, ou seja, há uma reciprocidade entre a aprendizagem motora e social. Dessa forma, dificuldades motoras podem influenciar diretamente nas relaçóes sociais de forma negativa, até mesmo nas relações familiares (LARKIN; SUMMERS, 2004). 
Além das dificuldades na vida diária, acadêmica e social, a criança com TDC apresenta dificuldades em atividades físicas como correr, saltar, pular corda, etc., podendo fazê-la desistir dessa prática, interferindo também nos níveis de condicionamento quando atingem a adolescência e a vida adulta. A inatividade leva a um baixo condicionamento físico, incluindo a performance aeróbica e anaeróbica, levando a um aumento de sua massa corporal e consequente obesidade (LARKIN; SUMMERS, 2004; DECONINCK et al., 2007).

Coetzee e Pienaar (2010), identificaram também problemas na perseguição visual em crianças com TDC e, um ano mais tarde, Coetzee; Pienaar (2011), concluíram que existe uma relação entre o controle de funçóes do músculo ocular e as crianças classificadas com TDC, sendo que crianças que apresentam desvios visuais têm o desempenho acadêmico e o desenvolvimento das habilidades motoras prejudicados.

Contudo, segundo Martini (2004), ainda não há um consenso quanto às razóes pelas quais crianças com TDC apresentam dificuldades na realização de tarefas psicomotoras.

\subsection{Possibilidades De INTERVENÇão PELA ATIVIDADE FísICA}

Atividades físicas regulares e brincadeiras durante a infância podem contribuir para uma melhora do desenvolvimento físico e social, assim como o conhecimento dos pais pode contribuir para o desenvolvimento global da criança. Sem esse conhecimento, a criança pode ser privada do suporte necessário ao seu desenvolvimento ideal (LARKIN; SUMMERS, 2004).

Em relação aos professores de Educação Física, a ênfase deve repousar sobre o encorajamento, diversão, esforço e participação ao invés da proficiência. Atividades em jogos onde a meta é a própria performance e não a da outra criança podem auxiliar, assim como trabalhar em pequenos grupos onde as habilidades exigidas enfrentarão poucos obstáculos. Modificar os equipamentos pode reduzir os riscos de lesão e materiais como bolas maiores ou macias podem ser estratégias efetivas. Mais do que isso, "Educadores Físicos podem desempenhar um papel crítico na identificação e avaliação deste transtorno do desenvolvimento da função motora nos alunos, bem como na implementação de uma intervenção" (CLARK et al., 2005, p. 49).

Para Clark et al. (2005), os professores de Educação Física podem considerar-se colaboradores essenciais na identificação do TDC, pois são treinados para observar e avaliar o movimento (são especialistas em comportamento e aprendizagem motora), uma vez que são eles que monitoram os alunos nas mais variadas e complexas situaçóes de movimento, sendo, portanto, mais precisos nessa identificaçáo do que professores de sala de aula.

Missiuna et al. (2004) sugerem o uso da estratégia "M.A.T.C.H.”, que visa adequar as atividades de acordo com as necessidades da criança ("M.A.T.C.H." each task to the needs of the child). M - Modify the task (modificar a tarefa), A - Alter yours expectations (alterar suas expectativas), $\mathrm{T}$ - Teach strategies (ensinar estratégias), $\mathrm{C}$ - Change the environment (mudar o ambiente) e $\mathrm{H}$ - Help by understanding (ajudar através da compreensão).

Para Schoemaker et al. (2003) e Rivard et al. (2007), é possível que professores de sala de aula também sejam capazes de identificar alunos com TDC, já que habilidades motoras também são exigidas nesse espaço. Missiuna et al. (2004), sugere que em sala de aula reduza-se o conteúdo escrito e permita-se tempo extra para completar as tarefas escritas, sendo importan- 
te diminuir a parte motora da tarefa, mas sem diminuir as expectativas cognitivas. Por exemplo, as crianças podem desenhar a história ao invés de escrevê-la.

Hillier (2010), estudou o efeito de um programa de terapia aquática para crianças com TDC, afirmando que essa intervenção pode auxiliar na estabilização dos músculos e aprimorar as habilidades motoras e a coordenação motora grossa, desde que as metas e os resultados sejam individuais devido à falta de evidências mais precisas.

No Brasil, Silva et at. (2011) estudaram o efeito de um programa de intervenção motora para escolares com indicativo de TDC. As autoras verificaram que houve uma melhora no desempenho motor das crianças após a proposta de intervenção, evidenciando-se os benefícios da prática motora. Concluíram, dessa forma e com base em outros estudos dessa natureza, que o movimento tem um grande valor na vida das crianças, destacando a necessidade e a importância do desenvolvimento de projetos que visem desenvolver e potencializar as habilidades dos escolares com dificuldades motoras.

Para Larkin e Summers (2004), além de estratégias educacionais, políticas públicas são necessárias para garantir o acesso dessas crianças em programas de atividades físicas em ambientes socialmente favoráveis.

\section{Conclusão}

Crianças com TDC representam uma parcela significativa da população em idade escolar. Elas apresentam dificuldades motoras que impactam diretamente em suas atividades diárias em casa, na escola e no lazer. Esse transtorno pode atingir uma elevada parcela das crianças em idade escolar e essas crianças geralmente têm dificuldades em se relacionar socialmente, afetando também a capacidade de aprender e a atenção.

Contudo, crianças com TDC podem ser reconhecidas pelos professores de diferentes disciplinas, em especial, pelos professores de Educação Física através de tarefas informais e, assim, podem organizar uma intervenção apropriada. Assim, tanto os professores como os pais podem se tornar parceiros das crianças colaborando para seu desenvolvimento global.

De acordo com o estudo realizado, tem sido evidenciada a relação entre os comportamentos sociais e o TDC no que se refere ao comprometimento do cumprimento efetivo das atividades cotidianas da criança implicando em desajustes sociais. Como medidas de intervenção, foram desenvolvidas propostas que envolvem atividades físicas personalizadas que enfatizem treinos com maior tempo de execução.

Os estudos enfatizam a presença dos profissionais que atuam com atividades físicas no que se refere à sensibilização dos mesmos para perceber as características das crianças que apresentam TDC, bem como para aplicar instrumentos que permitam identificar possíveis distorçóes na coordenação da criança. 


\section{REFERENCIAS}

ARAÚJO, C.R.S. Efeitos da terapia motora cognitiva no desempenho de atividades de crianças com transtorno do desenvolvimento da coordenação. 2010. 97f. Dissertação (Mestrado em Ciências da Reabilitação) - Escola de Educação Física, Fisioterapia e Terapia Ocupacional, Universidade Federal de Minas Gerais, Belo Horizonte. Disponível em:<http://www.dominiopublico.gov.br>. Acesso em: 18 ago. 2013.

ASSOCIAÇÃO AMERICANA DE PSIQUIATRIA (American Psychiatric Association - APA). DSMIV - Manual diagnóstico e estatístico de transtornos mentais. Tradução de Cláudia Dornelles. 4.ed. Porto Alegre: Artmed, 2002.

BARNHART, R. C. et al. Developmental coordination disorder. Physical Therapy, v.83, n.8, p.722731, 2003.

BONIFACCI, P. Children with low motor ability have lower visual-motor integration ability but unaffected perceptual skills. Human Movement Science, v.23, p.157-168, 2004.

CERMAK, S. et al. What is developmental coordination disorder? In: CERMAK, S.; LARKIN, D. Developmental coordination disorder. Clifton Park: Delmar, 2002.

CHENG, H.C. et al. Reading and writing performances of children 7-8 years of age with developmental coordination disorder in Taiwan. Research in Developmental Disabilities, v.32, p.25892594, 2011.

CLARK, J. E. et al. Developmental coordination disorder: issues, identification, and intervention. Joperd, v.76, n.4, p.49-53, 2005.

COETZEE, D.; PIENAAR, A. E. The nature and scope of ocular muscle control deviations among 7 to 8 year-old children diagnosed with developmental coordination disorder (DCD). African Journal for Physical, Health Education, Recreation and Dance, v.17, n.4, p.887-901, 2011.

COETZEE, D.; PIENAAR, A. E. The role of visual functions in persisting developmental coordination disorder (DCD) among 7-year-old children: a follow-up study. African Journal for Physical, Health Education, Recreation and Dance, v.16, n.2, p.251-264, 2010.

COUTINHO, M.T.C. et al. Transtorno do desenvolvimento da coordenação: prevalência e dificuldades motoras de escolares da cidade de Porto Alegre. In: CONBRACE, 17.; CONICE, 4., 2011, Porto Alegre. Anais .... Porto Alegre: ESEF/UFRGS, 2011. Disponível em <http://http://www. rbceonline.org.br/congressos/index.php/XVII_CONBRACE/2011/paper/view/3292>. Acesso em: 19 abr. 2014.

EMCK, C. et al. Gross motor performance and self-perceived motor competence in children with emotional, behavioural, and pervasive developmental disorders: a review. Developmental Medicine \& Child Neurology, v.51, p.501-517, 2009.

DECONINCK, F. J. A. et al. Differences in gait between children with and without developmental coordination disorder. Motor Control, v.10, p.125-142, 2006.

DECONINCK, F. J. A. et al. Sensory contributions to balance in boys with developmental coordination disorder. Adapted Physical Activity Quarterly, v.25, p.17-35, 2007.

FAUGHT, B. E. et al. Screening for motor coordination challenges in children using teacher ratings of physical ability and activity. Human Movement Science, v.27, p.177-189, 2008.

FILIPCIC, T.; OZBIC, M. Prediction of learning difficulties with the test of complex imitation of movement. Acta Universitatis Palackianae Olomucensis Gymnica, v.38, n.4, p.25-29, 2008. 
FRANÇA, C. Desordem Coordenativa Desenvolvimental em crianças de 7 e 8 anos de idade. 2008. 95f. Dissertação (Mestrado em Ciências do Movimento Humano) - Centro de Ciências da Saúde e do Esporte, Universidade do Estado de Santa Catarina, Florianópolis, 2008. Disponível em: $<$ http://www. dominiopublico.gov.br>.Acesso em: 18 ago. 2013.

GEUZE, R. H. Static balance and developmental coordination disorder. Human Movement Science, v.22, p.527-548, 2003.

GIBBS, J. et al. Dyspraxia or developmental coordination disorder? Unravelling the enigma. Archives of Disease in Childhood, v. 92, p. 534-539, 2007.

GOODGOLD-EDWARDS, S.A.; CERMAK, S.A. Integrating motor control and learning concepts with neuropsychological perspectives on apraxia and developmental dyspraxia. American Journal of Occupational Therapy, v.44, n.5, p.431-439, 1990.

HENDERSON, S.E.; SUGDEN, D.A. Movement assessment battery for children manual. London: The Psychological Corporation, 1992.

HILLIER, S. Aquatic physical therapy for children with developmental coordination disorder: A pilot randomized controlled trial. Physical \& Occupational Therapy in Pediatrics, v.30, p.111-124, 2010.

IVERSEN, S. et al. Intervention for 6-year-old children with motor coordination difficulties: parental perspectives at follow-up in middle childhood. Advances in Physiotherapy, v.7, p.67-76, 2005.

JOHNSON, D. C.; WADE, M. G. Judgment of action capabilities in children at risk for developmental coordination disorder. Disability and Rehabilitation, v. 29, p.33- 45, 2007.

LARKIN, D.; SUMMERS, J. Implications of movement difficulties for social interaction, physical activity, play, and sports. In: DEWEY, D.; TUPPER, D.E. (Eds.). Developmental motor disorders: a neuropsychological perspective. The Guilford Press: New York, 2004.

LLOYD, M. et al. Self-regulation of sport specific and educational problem-solving tasks by boys with and without DCD. Adapted Physical Activity Quarterly, v.23, p.370-389, 2006.

MAGALHÁES, L. C. et al. Activities and participation in children with developmental coordination disorder: a systematic review. Research in Developmental Disabilities, v.32, p.1309-1316, 2011.

MANDICH, A.; POLATAJKO, H.J. Developmental coordination disorder: Mechanisms, measurement and management.Human Movement Science, v.22, n.4/5, p.407-411, 2003.

MARTINI, R. Metacognitive processes underlying psychomotor performance in children with differing psychomotor abilities. Adapted Physical Activity Quarterly, v. 21, p. 248-268, 2004.

MAY-BENSON, T. et al. Daily living skills and developmental coordination disorder. In: CERMAK, S.; LARKIN, D. (Eds.). Developmental coordination disorder. Clifton Park: Delmar, 2002.

MISSIUNA, C. et al. They're bright but can't write: developmental coordination disorder in school aged children. Teaching Exceptional Children Plus, v.1, n.1, 2004.

MON-WILLIAMS, M. et al. The preparation of reach-to-grasp movements in adults, children, and children with movement problems. The Quarterly Journal of Experimental Psychology, v.58, p.1249$1263,2005$.

OZBIC, M.; FILIPCIC, T. Complex imitation of gestures in school-aged children with learning difficulties. Kinesiology, v.42, n.1, p.44-55, 2010. 
POLATAJKO, H.J.; CANTIN, N. Developmental coordination disorder (dyspraxia): an overview of the state of the art. Seminars in Pediatric Neurology, v.12, n.4, p.250-258, 2006.

POOLE, J.L. et al. The mechanisms for adult-onset apraxia and developmental dyspraxia: an examination comparison of error patterns. American Journal of Occupational Therapy, v.51, n.5, p.339346, 1997.

RIVARD, L. M. et al. Understanding teachers' perceptions of the motor difficulties of children with developmental coordination disorder (DCD). British Journal of Educational Psychology, v.77, p.633648, 2007.

ROSA NETO, F. Manual de avaliação motora. Porto Alegre: Artmed, 2002.

SANGER, T.D. et al. Definition and classification of negative motor signs in childhood. Pediatrics, v.118, p.2159-2167, 2006.

SCHOEMAKER, M. M. et al. Psychometric properties of the Movement Assessment Battery for children-checklist as a screening instrument for children with a developmental co-ordination disorder. British Journal of Educational Psychology, v.0, p.425-441, 2003.

SILVA, E.V.A. et al. Programa de intervenção motora para escolares com indicativo de transtorno do desenvolvimento da coordenação - TDC. Revista Brasileira de Educação Especial, Marília, v.17, n.1, p.137-150, 2011.

STRACKER, L. M. et al. Rationale, design and methods for a randomized and controlled trial of the impact of virtual reality games on motor competence, physical activity, and mental health in children with developmental coordination disorder. BMC Public Health, v.11, p.654-666, 2011.

ULRICH, D. A. Test of gross motor development - second edition. Austin: Pro-Ed., 2000.

WAELVELDE, H.V. Developmental coordination disorder: a developmental delay? Palestra, v.23, n.1, p. 57-59, 2007.

WERNER, J.M. et al. Neural correlates of developmental coordination disorder: the mirror neuron system hypothesis. Journal of Behavioral and Brain Science, v. 2, p.258-268, 2012.

WILSON, P. H. Practitioner review: approaches to assessment and treatment of children with DCD: an evaluative review. Journal of Child Psychology and Psychiatry, v.46, p.806-823, 2005.

WILSON, B. N. et al. Psychometric properties of the revised developmental coordination disorder questionnaire. Physical \& Occupational Therapy in Pediatrics, v.29, p.184-204 2009.

WUANG, Y. P. et al. Profiles and cognitive predictors of motor functions among early school-age children with mild intellectual disabilities. Journal of Intellectual Disability Research, v.52, p.1048-1060, 2008 .

ZWICKER, J. G. Neural correlates of developmental coordination disorder: a review of hypotheses. Journal of Child Neurology, v.24, n.10, p.1273-1281, 2009.

ZWICKER, J.G. et al. Developmental coordination disorder: a review and update. European Journal of Pediatric Neurology, v.16, p.573-581, 2012.

Recebido em: 12/11/2014

Reformulado em: 15/05/2015

Aprovado em: 16/05/2015 